\title{
Clinical Effect of Doxycycline Combined with Compound Sulfamethoxazole and Rifampicin in the Treatment of Brucellosis Spondylitis
}

\author{
Xin-Ming Yang (DD \\ Yong-Li Jia \\ Ying Zhang \\ Pei-Nan Zhang \\ Yao Yao \\ Yan-Lin Yin \\ Ye Tian
}

Department of Orthopaedics, The First Affiliated Hospital of Hebei North University, Zhangjiakou, 075000, Hebei, People's Republic of China
Correspondence: Xin-Ming Yang Department of Orthopaedics, The First Affiliated Hospital of Hebei North University, No. 12, Changqing Road, Qiaoxi District, Zhangjiakou, 075000, Hebei, People's Republic of China Tel +86 3। 3-8046926

Email yxmI I20@sohu.com
Objective: The purpose of this study was to determine the clinical value of triple antibiotic therapy consisting of doxycycline, compound sulfamethoxazole and rifampicin in the treatment of brucellosis spondylitis.

Methods: A retrospective analysis was performed on 100 patients with brucellosis spondylitis admitted to the First Affiliated Hospital of Hebei North University from March 2016 to June 2019. Patients were divided into the following two groups: the control group $(n=50)$ treated with dual antibiotic therapy (rifampicin + compound sulfamethoxazole), and the observation group $(n=50)$ treated with triple antibiotic therapy (rifampicin + doxycycline + compound sulfamethoxazole). The treatment effect, low back pain relief, levels of erythrocyte sedimentation rate (ESR), procalcitonin (PCT) and C-reactive protein (CRP), as well as the adverse reactions were compared between the two groups.

Results: The response rate of the observation group was significantly higher than that of the control group $(P<0.05)$. Before treatment, there was no significant difference in the low back pain assessed by the visual analogue scale (VAS), or levels of ESR, PCT and CRP between the two groups $(P>0.05)$. But after treatment, the VAS score and the levels of ESR, PCT and CRP in observation group were lower than those in the control group $(P<0.05)$. No significant difference was found in the incidence of adverse reactions $(P>0.05)$.

Conclusion: The triple antibiotic therapy of doxycycline, compound sulfamethoxazole and rifampicin is effective in the treatment of brucellosis spondylitis. It can significantly alleviate patients' back pain and inflammation with a high safety profile, which is worthy of clinical application.

Keywords: doxycycline, compound sulfamethoxazole, rifampicin, Brucella, spondylitis

Brucellosis spondylitis, abbreviated as brucellosis, is a zoonotic contagious disease caused by Brucella infection. When Brucella infection invades the human body, it travels through the bloodstream to various organs in the body. When it invades the spine, it is clinically called Brucellar spondylitis, or brucellosis spondylitis. ${ }^{1-3}$ In the late 1970s and 1980s, the prevalence of this disease tended to ease. However, recent years have witnessed a gradual increase in the number of patients with brucellosis along with the development of animal husbandry, with an incidence of $2 \%$ to $53 \%$. $^{4-6}$ Inflammation caused by brucellosis often involves the spinal cord and nerve roots, leading to neurological symptoms and even paraplegia in severe cases. $^{5,7}$ Currently, antibiotics are an important treatment for the disease, and dual or even triple antibiotic therapy is often used in clinical treatment. Therefore, the 
choice of antibiotics in clinical practice should be more cautious. ${ }^{89}$ In this study, the clinical data and treatment status of 100 patients admitted to our hospital from March 2016 to June 2019 were retrospectively analyzed. The report is as follows.

\section{Materials and Methods}

\section{General Information}

A retrospective analysis was performed on 100 patients with brucellosis spondylitis admitted to the First Affiliated Hospital of Hebei North University from March 2016 to June 2019. Patients treated with dual antibiotic therapy (rifampicin + compound sulfamethoxazole) were included in the control group ( $\mathrm{n}=50)$, and those treated with triple antibiotic therapy (rifampicin + doxycycline + compound sulfamethoxazole) were assigned to the observation group $(\mathrm{n}=50)$. Inclusion criteria: (1) All patients had a history of exposure to cattle and sheep to varying degrees or a history of eating undercooked beef and mutton or drinking unpasteurized dairy products. (2) All patients have a slow onset, accompanied by flaccid hypothermia (body temperature $<38.5^{\circ} \mathrm{C}$ ), night sweats and fatigue; (3) Diagnosis of brucellosis spondylitis based on medical history and imaging; (4) The indications for surgical treatment were not reached. Exclusion criteria: (1) Patients Aged less than 18 or more than 70, or those during pregnancy or lactation; (2) Those with allergies to the study medication; (3) Those with serious primary diseases such as cardiovascular, cerebrovascular, liver, kidney or hematopoietic system; (4) Patients with mental illness; (5) Those who cannot take medications on time as required, or terminated treatment by themselves; (6) Those with incomplete medical records. The general data were comparable between the two groups, with no significant difference in gender, age, course of disease, lesion location and bacterial classification $(P>0.05)$, as shown in Table 1. This study was conducted in accordance with the amended Declaration of Helsinki and was approved by the Ethics Committee of the First Affiliated Hospital of Hebei North University, all subjects signed an informed consent form.

\section{Laboratory Inspection}

The Brucella agglutination test results showed positive, and the erythrocyte sedimentation rate (ESR) increased to 32$93 \mathrm{~mm} / \mathrm{h}$. The results of blood routines were within the reference range, with the C-reactive protein (CRP) of 28 $65 \mathrm{mg} / \mathrm{L}$ and the procalcitonin (PCT) of $2.43-4.65 \mu \mathrm{g} / \mathrm{L}$.
Table I Comparison of General Information Between the Two Groups

\begin{tabular}{|l|l|l|l|l|}
\hline Grouping & $\begin{array}{l}\text { Control } \\
\text { Group }(\mathbf{n}= \\
\mathbf{5 0})\end{array}$ & $\begin{array}{l}\text { Observation } \\
\text { Group }(\mathbf{n}= \\
\mathbf{5 0}\end{array}$ & $\mathbf{t} / \mathbf{X}^{\mathbf{2}}$ & $\mathbf{P}$ \\
\hline Age (years) & $43.56 \pm 5.23$ & $42.97 \pm 5.02$ & 0.023 & 0.67 \\
\hline $\begin{array}{l}\text { Gender [n(\%)] } \\
\text { Male } \\
\text { Female }\end{array}$ & $\begin{array}{l}29(58.0) \\
21(42.0)\end{array}$ & $\begin{array}{l}27(54.0) \\
23(46.0)\end{array}$ & 0.16 & 0.69 \\
\hline $\begin{array}{l}\text { Course of disease } \\
\text { (months) }\end{array}$ & $37.82 \pm 8.32$ & $38.11 \pm 8.01$ & 0.036 & 0.58 \\
\hline $\begin{array}{l}\text { Bacterial typing } \\
\text { Br. bovis } \\
\text { Br. melitensis }\end{array}$ & $\begin{array}{l}27(54.0) \\
23(46.0)\end{array}$ & $\begin{array}{l}28(56.0) \\
22(44.0)\end{array}$ & 0.04 & 0.84 \\
\hline $\begin{array}{l}\text { Regions } \\
\text { L3-4 } \\
\text { L4-5 } \\
\text { L5-SI }\end{array}$ & $\begin{array}{l}18(36.0) \\
20(40.0) \\
12(24.0)\end{array}$ & $\begin{array}{l}17(34.0) \\
22(44.0) \\
11(22.0)\end{array}$ & 0.18 & 0.92 \\
\hline
\end{tabular}

\section{Imaging Manifestations}

GE Lightspeed Ultra multislice CT Scanner was used for routine scanning; GE portable $\mathrm{C}$-arm X-ray machine was used for fluoroscopy examination; GE Signa HD Highfield magnetic resonance imaging (MRI) was used to scan conventional spin echo (SE) sequences at $\mathrm{T} 1$ weighted image (T1WI), T2 weighted image (T2WI), short-TI inversion-recovery (STIR), as well as horizontal T1WI and T2WI. X-ray films demonstrated varying degrees of narrowing of the intervertebral-disc space. The vertebral body showed worm-eaten damage, with bone hyperplasia at the edge and some bone bridges. CT showed multiple small lesions in the vertebral body, most of which were located at the edge of the centrum, with osteosclerosis at the edge of the lesion. MRI showed low signal intensity on T1WI, high signal intensity on T2WI, uneven signal intensity of intervertebral disc invasion, and intervertebral space stenosis. Abscesses can be seen on both sides of the vertebral body with ill-defined boundaries. The scanning images can be found in Figure 1.

\section{Treatment Methods}

Both groups of patients received routine care and were instructed to drink more water and take vitamin $\mathrm{B} / \mathrm{C}$ and nutrients. Patients with fever were given physical hypothermia or antipyretic drugs. For those with pain, they were given analgesics and told to rest. All patients were treated 
A

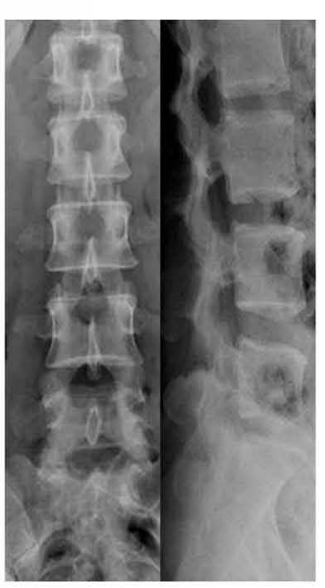

B

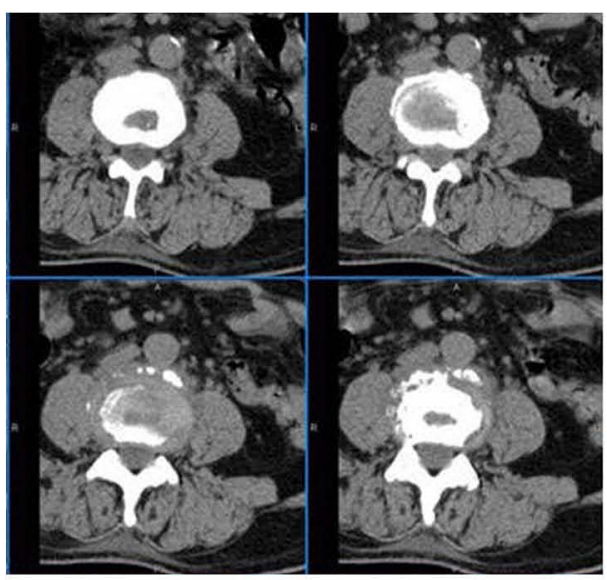

C

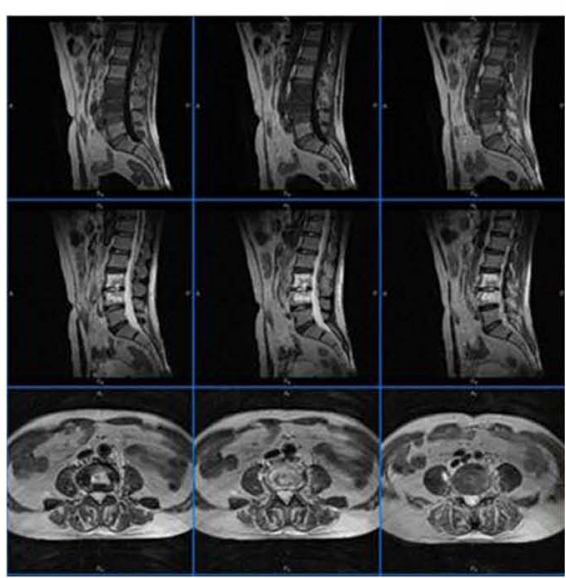

Figure I Imaging manifestations of a typical case of brucellosis spondylitis. Anteroposterior X-ray: Visible vertebral bone hyperplasia, sclerosis, irregular worm-like damage, narrowing of the intervertebral space, ossification of the anterior and posterior longitudinal ligaments of the spine, as well as narrowing and blurring of the facet joint space (A); CT: bone destruction can be seen, which are multiple round, quasi-round, or patchy low-density foci with obvious hyperplastic sclerosis bands around (B); MRI: it can be seen that the vertebral body bone and the surrounding soft tissues have abnormal signals, the intervertebral space is narrow, and the vertebral body shows uneven signal. TIWI takes on low signal, but $\mathrm{T} 2 \mathrm{WI}$ high signal (C).

with a combination regimen. Patients in the control group $(\mathrm{n}=50)$ were treated with dual antibiotic therapy composed of rifampicin (Manufacturer: Shenyang Shuangding Pharmaceutical Co. Ltd.; SFDA Approval Number: H20050725; Specifications: 5mL: 0.3g (rifampicin)*5) and compound sulfamethoxazole (Manufacturer: Shandong Fangming Pharmaceutical Group Co. Ltd.; SFDA Approval Number: H37023306; Specifications: 2mL: $0.4 \mathrm{~g} * 10$ injection). Administration: rifampicin: $0.45 \mathrm{~g} /$ time, qd, intravenous infusion; compound sulfamethoxazole: $0.1 \mathrm{~g} /$ time, qd, intravenous infusion. The observation group $(n=50)$ was treated by triple antibiotic therapy, which consisted of rifampicin, doxycycline, and compound sulfamethoxazole. Administration: doxycycline (Manufacturer: Hainan Tongkangli Pharmaceutical Co. Ltd.; SFDA Approval Number: H20060405; Specifications: 0.1g injection): $0.1 \mathrm{~g} /$ time, qd, intravenous infusion; the usage of rifampin and compound sulfamethoxazole is the same as described above. After the Brucella agglutination test indicating negative, patients continued to take the drugs for 2 weeks for a total of 1 to 2 courses. Both groups were prescribed 7 days a treatment period. If the Brucella agglutination test was negative after 2 treatment periods, the medication was continued for 2 weeks. In the treatment process, patients with high fever were given physical cooling, antipyretic and analgesic drugs, and those with low back pain were given non-steroidal anti-inflammatory drugs. At the same time, the liver and kidney function was tested, and liver protection treatment was given if necessary.
In addition, patients were asked to stay in bed and enhance nutritional support.

\section{Evaluation Criteria}

(1) Curative effect: Cured: During the follow-up which lasted for more than 6 months, the clinical symptoms and signs of patients disappeared, with no fever, normal ESR, PCT and CRP levels, as well as spinal function recovery and bone healing as indicated by X-ray. Improved: no fever, and significant improvement in clinical symptoms and signs; ESR, PCT and CRP levels were close to normal, and the spinal function was significantly restored; X-ray showed that the bone is basically healed. Ineffective: Fever was still present, and the clinical symptoms and signs were not significantly improved; ESR, PCT and CRP levels were elevated, and the spinal function was not significantly recovered; X-ray showed bone healing failure, with positive Brucella agglutination test results or only a short period of symptom improvement. Effective rate $=($ cure + improvement $)$ cases/total number of cases $\times 100 \%$.

(2) The visual analogue scale (VAS) was used to assess the low back pain relief of patients. The higher the score, the intense the pain. ${ }^{10}$

(3) The serum levels of inflammatory indexes (ESR, PCT and CRP) before and after treatment were recorded and compared between the two groups. PCT was measured by the immunofluorescence method, and ESR and CRP were measured using an automatic biochemical analyzer. 
(4) Incidence of adverse reactions: Adverse reactions including gastrointestinal reactions (nausea and vomiting, abdominal pain and diarrhea), skin rash, liver and kidney damage, were recorded in both groups.

\section{Statistical Analysis}

SPSS 22.0 statistical software and GraphPad Prism 8.0 software were used for data statistical analysis and image rendering, respectively. $\mathrm{P}<0.05$ was considered to be statistically significant. Counting data were expressed as number of cases/percentages [n (\%)], and compared by the Chi-square test between groups. Measurement data were expressed as mean \pm standard deviation (mean $\pm \mathrm{SD}$ ); Independent sample $t$-test was used for inter-group comparison of measurement data, and paired $t$-test was used for intra-group comparison before and after treatment.

\section{Results}

\section{Comparison of General Information Between the Two Groups}

There were no significant differences in age, gender, course of disease, body mass index (BMI) or location of disease between the two groups $(P>0.05)$, as shown in Table 1 .

\section{Comparison of Treatment Efficacy Between the Two Groups}

In the observation group, 15 cases were cured, 27 cases were improved and 8 cases were ineffective, with an effective rate of $84 \%$; While the cured, improved and ineffective cases in the control group were 10, 23 and 17 respectively, and the effective rate was $66 \%$; The response rate of the observation group was higher than that of the control group $(P<0.05)$, as shown in Table 2 .

Table 2 Comparison of Treatment Efficacy Between the Two Groups (n,\%)

\begin{tabular}{|c|c|c|c|c|}
\hline Grouping & $\begin{array}{l}\text { Cured } \\
{[n(\%)]}\end{array}$ & $\begin{array}{l}\text { Improved } \\
\text { [n(\%)] }\end{array}$ & $\begin{array}{l}\text { Ineffective } \\
{[n(\%)]}\end{array}$ & $\begin{array}{l}\text { Effective } \\
\text { Rate (\%) }\end{array}$ \\
\hline $\begin{array}{l}\text { Observation group } \\
(n=50)\end{array}$ & $15(30.0)$ & $27(54.0)$ & $8(16.0)$ & $84 \%$ \\
\hline $\begin{array}{l}\text { Control group }(\mathrm{n}=50) \\
X^{2} \\
P\end{array}$ & $10(20.0)$ & $23(46.0)$ & $17(34.0)$ & $\begin{array}{l}66 \% \\
25.84 \\
<0.001\end{array}$ \\
\hline
\end{tabular}

\section{Comparison of VAS Score Between the} Two Groups

Before treatment, there was no significant difference in VAS score between the two groups $(P>0.05)$. After treatment, the VAS score of the observation group was significantly lower than that of the control group $(P<0.05)$, as shown in Figure 2.

\section{Comparison of Serum Inflammatory Indexes Between the Two Groups}

The two groups showed similar serum levels of PCT, CRP and ESR before treatment. However, the levels of serum PCT, CRP and ESR in the observation group were significantly lower than those in the control group after treatment $(P<0.05)$, as shown in Figure 3.

\section{Comparison of Adverse Reactions Between the Two Groups}

In the control group, there were 5 cases of gastrointestinal reactions, 3 cases of skin rash, 3 cases of liver damage and 2 cases of kidney function damage, with a total adverse reaction rate of $26 \%$. In the observation group, gastrointestinal reactions, rash, liver damage and kidney damage occurred in 3 cases, 2 cases, 2 cases and 2 cases respectively, and the overall adverse reaction rate was $18 \%$. There was no difference in the incidence of adverse reactions between the two groups $(P<0.05)$, as shown in Table 3 .

\section{Discussion}

Brucellosis is found globally. In China, the disease mostly occurs in areas with large pastoral areas such as Inner Mongolia and Xinjiang, while it is sporadically distributed in other provinces. ${ }^{11}$ Brucella is a ball-shaped gramnegative bacterium, with 6 genera, including Br. bovis, Br. melitensis and $\mathrm{Br}$. suis, among which the former two were the most common species in China. ${ }^{12,13}$ Brucella infection in humans, with no sex predilection, is caused by human contact with infected animals, eating infected food or breathing air contaminated with Brucella. It is mainly transmitted to the human body through animals and is difficult to spread from person to person. Its pathogenesis is complex, mainly associated with bacteria and toxins. ${ }^{14}$ The main clinical symptoms during the acute phase of the disease are fever, hyperhidrosis, and joint pain. ${ }^{15}$ Improper treatment can lead to the progression of acute brucellosis to a chronic disease, complicated by osteoarthritis, neurological brucellosis, etc., seriously 


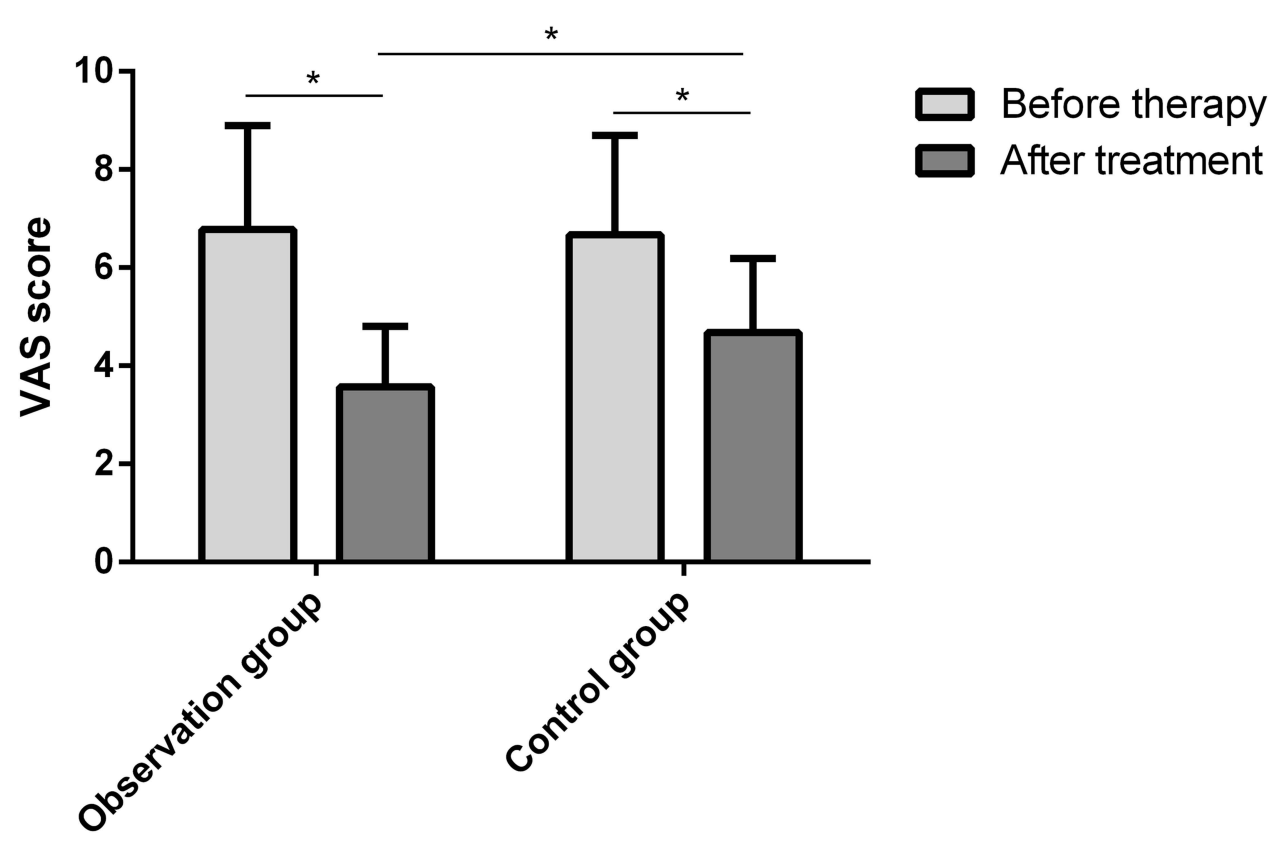

Figure 2 VAS scores of the two groups before and after treatment. Note: $* \mathrm{P}<0.05$.
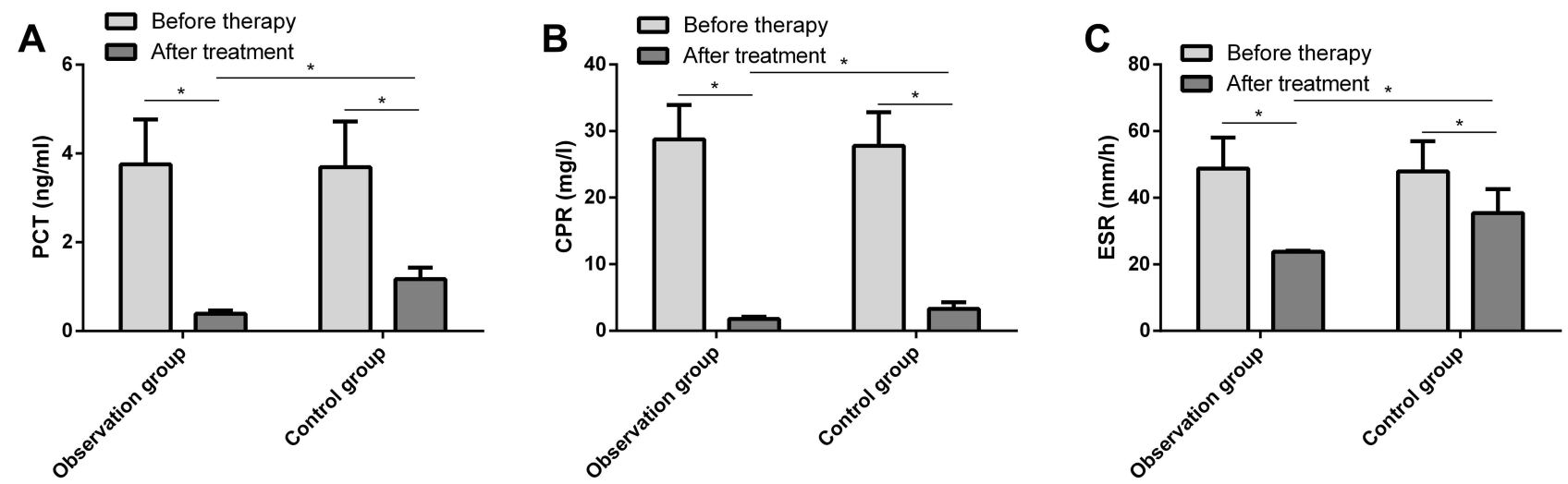

Figure 3 Comparison of serum inflammatory indexes between the two groups. Comparison of serum PCT (A), CRP (B) and ESR (C) levels between the two groups of patients before and after treatment.

Note: $* \mathrm{P}<0.05$

affecting the quality of life of patients. ${ }^{16}$ In general, after Therefore, the choice of antibiotics, the course of treatdiagnosis and standardized treatment, patients have ment and patient compliance are critical to the treatment favourable outcomes and low disease recurrence. and prognosis of the disease.

Table 3 The Occurrence of Adverse Reactions in the Two Groups of Patients (n, \%)

\begin{tabular}{|l|l|l|l|l|l|}
\hline Grouping & $\begin{array}{l}\text { Gastrointestinal } \\
\text { Reactions [n(\%)] }\end{array}$ & $\begin{array}{l}\text { Skin Rashes } \\
{[\mathbf{n}(\%)]}\end{array}$ & $\begin{array}{l}\text { Liver Damage } \\
{[\mathbf{n}(\%)]}\end{array}$ & $\begin{array}{l}\text { Kidney Function } \\
\text { Damage [n(\%)] }\end{array}$ & $\begin{array}{l}\text { Total Incidence } \\
(\%)\end{array}$ \\
\hline Control group $(\mathrm{n}=50)$ & $5(10.0)$ & $3(6.0)$ & $3(6.0)$ & $2(4.0)$ & $26 \%$ \\
\hline $\begin{array}{l}\text { Observation group }(\mathrm{n}=50) \\
X^{2}\end{array}$ & $3(6.0)$ & $2(4.0)$ & $2(4.0)$ & $2(4.0)$ & $18 \%$ \\
P & & & & & 0.93 \\
\end{tabular}


Brucellosis spondylitis is one of the specific infectious diseases. Due to Brucella infection of the cones, the clinical symptoms of patients mainly include vertebral abscess, vertebral body destruction, and intervertebral disc inflammation, which in the long term, will cause spinal injury, leading to spine and even spinal cord lesions. ${ }^{17}$ According to "Guidelines for Diagnosis and Treatment of Mycosis" issued by the National Health Commission, doxycycline combined with rifampicin or streptomycin is recommended as the first-line drug therapy for brucellosis; The second-line drug therapy, ie, doxycycline in combination with sulfonamides or tobramycin or rifampicin in combination with fluoroquinolones, may be used as appropriate when first-line drugs cannot be combined or are not effective. ${ }^{18,19}$ Brucellosis spondylitis, characterized by changes in intervertebral disc inflammation, is one of the complications of brucellosis, with an incidence of up to $60 \%{ }^{20,21}$ In the early stage of the disease, conservative treatment with drugs is the mainstay of treatment. When the pain cannot be relieved by the conservative treatment, surgical treatment can be a option for those who meet the surgical indications. ${ }^{22-24}$

At present, brucellosis is mainly treated with antibiotics, including rifampicin, levofloxacin, doxycycline, and streptomycin. ${ }^{25,26}$ Clinically, antibacterial drugs that can enter cells are usually used in combination with other antibiotics to achieve drug penetration into macrophages, so as to improve the efficacy and prognosis. ${ }^{27}$ Both rifampicin and doxycycline are antibiotics that can enter cells. Rifampicin is a semi-synthetic broad-spectrum antibiotic of rifamycin, which has certain antibacterial activity against a variety of pathogenic microorganisms such as Gram-negative bacteria. Its mechanism of action is to inhibit the synthesis of RNA by bacteria and block transcription, thereby blocking the synthesis of protein and DNA and exerting its antibacterial effect; However, the effect of single drug is not ideal. ${ }^{28,29}$ Doxycycline, also an antibiotic with a broad antibacterial spectrum, has strong antibacterial activity against sensitive gram-positive cocci and gram-negative bacilli. It can quickly bind to $30 \mathrm{~S}$ ribosomal subunits and inhibit t-RNA binding to play its antibacterial effect. ${ }^{30,31}$ Sulfonamides are a kind of broad-spectrum antibacterial drugs, which are mainly used for the prevention and treatment of infectious diseases in clinical practice. In addition to their stability, they are widely used as feed additives or animal disease treatment drugs in veterinary clinics and animal husbandry. Sulfonamide antibiotics affect the synthesis of dihydrofolate and inhibit bacterial growth and reproduction by competing with sulfa drugs and receptors for dihydrofolate synthase. ${ }^{32,33}$ The results of this study showed that the response rate of the triple antibiotic therapy composed of doxycycline, compound sulfamethoxazole and rifampicin in the treatment of brucellosis spondylitis was $84 \%$, while that of the combined therapy of compound sulfamethoxazole and rifampicin was $66 \%$, indicating that the therapeutic effect of the triple antibiotic therapy is better than that of the dual therapy. In addition, it was found that there were no significant differences in the VAS score and serum levels of PCT, CRP and ESR between the two groups before treatment. After treatment, however, the above indexes in the observation group were lower than those in the control group, indicating that the triple antibiotic therapy (doxycycline + compound sulfamethoxazole + rifampicin) is more effective than the dual antibiotic therapy (compound sulfamethoxazole + rifampicin) in alleviating pain and reducing inflammation. With monotherapy, some patients may experience gastrointestinal reactions such as nausea and vomiting, as well as adverse reactions such as liver and kidney function damage, especially when antibiotics are used. ${ }^{34}$ Therefore, in the case of combined use of antibiotics, it is necessary to pay attention to and minimize the occurrence of adverse reactions. The results of this study showed that the total incidence of adverse reactions was $18 \%$ in the observation group and $26 \%$ in the control group, with no significant difference between the two groups. It suggests that mastering the use time and dose of doxycycline will help to control and reduce the adverse reactions of the combined use of antibiotics.

Due to the design of this study, it still has some limitations. The sample size may be too small to detect differences between the two groups. So, the similarities in clinical outcomes may be a type II error, as is evident from our study. Thus, a well-designed trial with prospective data collection and sample size calculation is needed to confirm the results obtained in this study and to demonstrate the clinical effect of doxycycline combined with compound sulfamethoxazole and rifampicin in the treatment of brucellosis spondylitis.

In summary, compared with dual antibiotic therapy of compound sulfamethoxazole and rifampicin, the triple antibiotic therapy consisting of doxycycline, compound sulfamethoxazole and rifampicin is more effective in treating brucellosis spondylitis. It has a more significant effect on reducing pain and inflammation in patients, with a relatively high safety profile, which is worthy of clinical application. 


\section{Acknowledgment}

I would like to express my gratitude to all those helped me during the writing of this thesis. I acknowledge the help of my colleagues, Ying Zhangand Yongli Jia who offered me suggestions in academic studies.

\section{Disclosure}

The authors report no conflicts of interest in this work.

\section{References}

1. Carugati M, Biggs HM, Maze MJ, et al. Incidence of human brucellosis in the Kilimanjaro Region of Tanzania in the periods 2007-2008 and 2012-2014. Trans $R$ Soc Trop Med Hyg. 2018;112(3):136-143. doi:10.1093/trstmh/try033

2. Yagupsky P, Morata P, Colmenero JD. Laboratory diagnosis of human brucellosis. Clin Microbiol Rev. 2019;33(1):e00073-00019. doi:10.1128/CMR.00073-19

3. Zhang N, Zhou H, Huang DS, Guan P. Brucellosis awareness and knowledge in communities worldwide: a systematic review and meta-analysis of 79 observational studies. PLoS Negl Trop Dis. 2019;13(5):e0007366. doi:10.1371/journal.pntd.0007366

4. Deng Y, Liu X, Duan K, Peng Q. Research progress on brucellosis. Curr Med Chem. 2019;26(30):5598-5608. doi:10.2174/ 0929867325666180510125009

5. Zheng R, Xie S, Lu X, et al. A systematic review and meta-analysis of epidemiology and clinical manifestations of human brucellosis in China. Biomed Res Int. 2018;2018:1-10. doi:10.1155/2018/5712920

6. Zhang N, Huang D, Wu W, et al. Animal brucellosis control or eradication programs worldwide: a systematic review of experiences and lessons learned. Prev Vet Med. 2018;160:105-115. doi:10.1016/j. prevetmed.2018.10.002

7. Khurana SK, Sehrawat A, Tiwari R, Prasad M, Chaicumpa W. Bovine brucellosis - a comprehensive review. Vet Q. 2021;41 (1):61-88. doi:10.1080/01652176.01652020.01868616

8. Bagheri Nejad R, Krecek RC, Khalaf OH, Hailat N, Arenas-Gamboa AM. Brucellosis in the Middle East: current situation and a pathway forward. PLoS Negl Trop Dis. 2020;14(5):e0008071. doi:10.1371/ journal.pntd.0008071

9. Jiang H, O'Callaghan D, Ding JB. Brucellosis in China: history, progress and challenge. Infect Dis Poverty. 2020;9(1):1-4 doi:10.1186/s40249-020-00673-8

10. Cline ME, Herman J, Shaw ER, Morton RD. Standardization of the visual analogue scale. Nurs Res. 1992;41(6):378-379. doi:10.1097/ 00006199-199211000-00013

11. Bouaziz MC, Ladeb MF, Chakroun M, Chaabane S. Spinal brucellosis: a review. Skeletal Radiol. 2008;37(9):785-790. doi:10.1007/ s00256-007-0371-x

12. Heavey E. Brucellosis: a global concern. Nursing. 2019;49(5):14-16. doi:10.1097/01.NURSE.0000554623.05347.a0

13. Sohrabi A, Hajia M. Brucellosis: the current situation in Iran and neighboring countries. Infect Disord Drug Targets. 2020;20 (5):763-764. doi:10.2174/1871526519666190923163010

14. Blasco JM, Moreno E, Moriyón I. Brucellosis. In: Veterinary Vaccines: Principles and Applications. Wiley Online Library; 2021:295-316.

15. Mirnejad R, Jazi FM, Mostafaei S, Sedighi M. Epidemiology of brucellosis in Iran: a comprehensive systematic review and meta-analysis study. Microb Pathog. 2017;109:239-247. doi:10.1016/j.micpath.2017.06.005

16. Moliner Lahoz FJ, Gonzalo S. [Family outbreak of brucellosis. The importance of epidemiological suspicion]. Rev Esp Salud Publica. 2020;94:e202012145. Spanish.
17. Liu R, Lyu J, Luo C, Wei M, Wan Y, Wang L. Investigation of human brucellosis cases in newly affected counties in Sichuan province, 2015-2017. Zhonghua Liu Xing Bing Xue Za Zhi= Zhonghua Liuxingbingxue Zazhi. 2020;41(3):412-416.

18. Bodenham RF, Lukambagire AS, Ashford RT, et al. Prevalence and speciation of brucellosis in febrile patients from a pastoralist community of Tanzania. Sci Rep. 2020;10(1):1-11. doi:10.1038/s41598020-62849-4

19. Ece İ, Epçaçan S, Bayhan Gİ, Türe M. Diastolic dysfunction in patients with brucellosis despite the absence of infective endocarditis. Cardiol Young. 2020;30(12):1840-1843. doi:10.1017/ S1047951120002930

20. Enkelmann J, Stark K, Faber M. Epidemiological trends of notified human brucellosis in Germany, 2006-2018. Int J Infect Dis. 2020;93:353-358. doi:10.1016/j.ijid.2020.02.019

21. Wang X, Huang M, Cui B. Analysis on brucellosis epidemiological characteristics in Hainan province. Zhonghua Liu Xing Bing Xue Za Zhi= Zhonghua Liuxingbingxue Zazhi. 2019;40(3): 350-353.

22. Jiang H, Kan B. Current status, progress and suggestions regarding prevention and control of brucellosis in China. Zhonghua Liu Xing Bing Xue Za Zhi= Zhonghua Liuxingbingxue Zazhi. 2020;41 (9):1424-1427.

23. de Souza Ribeiro Mioni M, Vicente AF, Peres MG, et al. Brucellosis prevalence in Brazilian slaughterhouses with different meat inspection systems. J Food Prot. 2018;81(7):1073-1078. doi:10.4315/0362028X.JFP-17-451

24. Subramaniam K, Ali U. Cellulitis in human brucellosis: an atypical presentation. Malays J Pathol. 2019;41(3):359-364.

25. Dong S, Jiang H, Wang L. Progress in research and practice of brucellosis surveillance in China. Zhonghua Liu Xing Bing Xue Za Zhi= Zhonghua Liuxingbingxue Zazhi. 2019;40(7):870-874.

26. Bakri FG, AlQadiri HM, Adwan MH. The highest cited papers in brucellosis: identification using two databases and review of the papers' major findings. Biomed Res Int. 2018;2018:1-10. doi:10.1155/2018/9291326

27. Peng C, Li Y-J, Huang DS, Guan P. Spatial-temporal distribution of human brucellosis in mainland China from 2004 to 2017 and an analysis of social and environmental factors. Environ Health Prev Med. 2020;25(1):1-14. doi:10.1186/s12199-019-0839-z

28. Dadar M, Tiwari R, Sharun K, Dhama K. Importance of brucellosis control programs of livestock on the improvement of one health. Vet Q. 2021;41(1):137-151. doi:10.1080/01652176.2021.1894501

29. Lu D, Zhou Y, Jing Z. Epidemiology of Brucellosis and why should we strengthen the awareness of Brucella endocarditis: clinical features, diagnosis, treatment and outcome. Zhonghua Xin Xue guan Bing Za Zhi. 2020;48(11):901-905.

30. Facciolà A, Palamara MA, D'Andrea G, et al. Brucellosis is a public health problem in southern Italy: burden and epidemiological trend of human and animal disease. J Infect Public Health. 2018;11 (6):861-866. doi:10.1016/j.jiph.2018.07.007

31. Olfatifar M, Hosseini SM, Shokri P, Khodakarim S, Khadembashi N, Pordanjani SR. How to improve the human brucellosis surveillance system in Kurdistan Province, Iran: reduce the delay in the diagnosis time. Epidemiol Health. 2020;42:e2020058.

32. Li M, Pei X, Zhang J, Li L. Asymptotic analysis of endemic equilibrium to a brucellosis model. Math Biosci Eng. 2019;16 (5):5836-5850. doi:10.3934/mbe.2019291

33. Yoshida GJ. Emergency response to occupational brucellosis in a pharmaceutical manufacturing enterprise. $J$ Occup Health. 2019;61(2):206-207. doi:10.1002/1348-9585.12015

34. Shehabi A, Shakir K, El-Khateeb M, Qubain H, Fararjeh N, Shamat ARA. Diagnosis and treatment of 106 cases of human brucellosis. $J$ Infect. 1990;20(1):5-10. doi:10.1016/S0163-4453(90) 92214-6 


\section{Publish your work in this journal}

Drug Design, Development and Therapy is an international, peerreviewed open-access journal that spans the spectrum of drug design and development through to clinical applications. Clinical outcomes, patient safety, and programs for the development and effective, safe, and sustained use of medicines are a feature of the journal, which has also been accepted for indexing on PubMed Central. The manuscript management system is completely online and includes a very quick and fair peer-review system, which is all easy to use. Visit http://www. dovepress.com/testimonials.php to read real quotes from published authors. 